Hydrol. Earth Syst. Sci. Discuss.,

https://doi.org/10.5194/hess-2020-435-AC4, 2020

(C) Author(s) 2020. This work is distributed under

the Creative Commons Attribution 4.0 License.

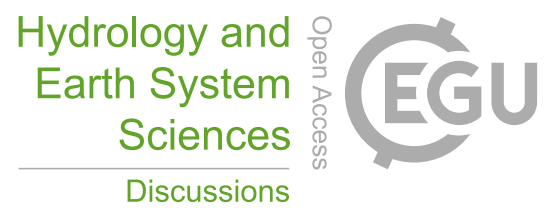

HESSD

Interactive

comment

\title{
Interactive comment on "Geophysically-based analysis of BTCs and ion exchange processes in soil" by Shany Ben Moshe et al.
}

Shany Ben Moshe et al.

benmoshe.shany@gmail.com

Received and published: 10 December 2020 


\section{Response to anonymous referee \#3}

We would like to thank anonymous referee $\# 3$ for the comments. We will account for them in a revised version of the paper, as we report in the following point-by-point reply:

\section{General comments (GC)}

GC 1 - The seemingly arbitrary consideration of only the partial real conductivity dataset need to be clearly addressed

Autors' response - The complete real conductivity signal (over the entire experiment) reflects the combination of the changes in pore-electrolyte conductivity due to the different processes that occur in the system over this time. Since the ADE modeling presented in the text reflects only the transport of the non-reactive ions, only the data of the initial BT was considered. We accept that this needs to be specifically clarified and will account for that in the revised version. Additionally, we intend to improve the ADE-modeling part of the paper by (a) simulating the non-reactive ion transport not only for $\mathrm{Cl}^{-}$but as a combination of all non-reactive species (in our system $\mathrm{Na}^{+}$and a fraction of the $\mathrm{Ca}^{2+}$ ions that behave as non-reactive solutes) and (b) present a model fit and prediction for the $\mathrm{Ca}^{2+}$ ions based on the SIP data of the 
secondary BT $(\mathrm{t}=0.9-2.5 \mathrm{~h})$. Transport parameters were originally included in $\mathrm{SI}$ and will now be presented in the main text body.

While the reviewer is correct that calibrating a model using SIP data would yield in a different set of parameters (compared to 'out-of-column' calibration). Yet, one may argue that with proper geomerical considerations the SIP-based calibration is superior (as it contains more information). Our intention is not necesarily to replace conventional calibration but to enhance it using more spatial data and exchange-related data

GC 2 -The interpretation of the imaginary conductivity time-series is rather brief and vague. The authors should combine their time-series analysis with an interpretation of imaginary conductivity spectra to improve the mechanistic description of the ion-exchange processes driving polarization signals.

Autors' response - We are not sure that we understand this comment properly. The imaginary conductivity results are discussed at length (starting from line 182). We directly connect the observed drop in the imaginary conductivity to the $\mathrm{Ca}^{2+}-\mathrm{Na}^{+}$exchange process and the ions' mobility. However, we agree that the discussion around the initial increase in $\sigma^{\prime \prime}$ (before it decreases) is not wide enough. It is worth noting in this context, that while similar patterns had been reported before (see Vaudelet et al., 2011), no explanation was suggested. We intend to expand the discussion around this part.

GC 3 - the introduction and discussion would both benefit from a comprehensive review and critical consideration of the current literature.

Autors' response - We fully accept this comment. The literature review inPrinter-friendly version cluded in the introduction will be expanded. For example, Izumoto et al., 2020, Slater et al., 2009 and Mellage et al., 2018 will be included as examples of previous studies that combined geo-electrical monitoring with transport processes or ADE modeling. 


\section{Specific comments (SC)}

SC 1 - Lines 35 - 54: The introduction should clearly define what "hydrogeophysics" is. The description of polarization mechanisms does not properly distinguish between EDL-polarization and membrane polarization and this should be clarified. Several of the statements should be properly and fairly referenced. In general the introduction would benefit from a more comprehensive review of the literature. Currently, the manuscript has a very short list of references.

Autors' response - We fully accept this comment. The introduction section of the revised version will include (as suggested by the reviewer) a definition of the term "hydrogeophysics" as well as a clear description of both EDL and the membrane polarization mechanisms and their contribution to the observed signal in soil systems. Additionally, we intend to significantly expend the literature review in the introduction and the discussion (see GC3).

SC 2 - Lines 56 - 60: Here and elsewhere, the manuscript should acknowledge previous studies that have combined breakthrough curve analysis in flow through systems with the monitoring of geoelectrical properties (e.g. Davis et al., 2006 and Deng et al., 2020) and combined these with transport models (e.g. Slater et al. 2009 and Mellage et al. 2018). How does the current work build on what has already been done? Similarly, the results of the current work should be compared to those in previous studies in the discussion.

Printer-friendly version

Autors' response - We fully accept this comment and will include proper reference to those (and other) works (see response to GC3).

Discussion paper 
SC 3 - Figure 3, Results: It seems as if only a subset of the real conductivity time series data were considered in the Hydrus-1D fitting scheme, and the cutoff was different at different channels (SIP measurement locations). The authors should clearly justify what data were used for model fitting. The authors should also report fitted parameter values. I would argue that a comparably good fit could have been achieved by considering only the Cl- breakthrough. However, the authors claim that real conductivity information could replace BTC analysis. Considering the full timeseries of real conductivity would likely yield a different parameter set than using only a subset of the data because of the ion exchange processes and associated signal changes later on. Thus, if only relying on SIP data for breakthrough curve analysis, most likely, a biased set of parameters would be estimated. This is contradictory to one of the main conclusions of the manuscript.

Autors' response - This comment is addressed in the 'General comments' section. Please see response to SC1.

SC 4 - Lines 196 - 200: The initial increase in the imaginary conductivity of the loam is not observed in the sand layer (in Figure 3b), however the drop in imaginary conductivity occurs in both the loam and sand layers. The authors should provide an explanation for this difference.

Autors' response - The initial increase in imaginary conductivity does occur for the sand layer as well (we assume that the reviewer intended to refer to Fig. 5b). This can be seen clearly in the attached figure below, which is an enlarged version of the sandy layer $\sigma^{\prime \prime}$ vs time graph.

SC 5 - Lines 200 - 204: Why would sodium-calcium exchange enhance ion mobility? This contradicts the interpretation of the decrease in imaginary conductivity. How would the inflowing solution and exchange process affect the interfacial/surface

Printer-friendly version

Discussion paper
Interactive

comment 
Autors' response - As presented in the methods section (SIP subsection), the complex conductivity is related to ion mobility in the EDL through the mobility, denoted as $\beta$. Imaginary conductivity changes have been shown to be related to the mobility of ions in the stern layer (e.g. Leroy et al, 2009, Vaudelet et al, 2011, Schwartz et al, 2012). As we explain in the text (line 186): "Adsorbed $\mathrm{Na}^{+}$ions maintain their hydration shell and hence, are weakly adsorbed to the soil and are more mobile compared to $\mathrm{Ca}^{2+}$. During the $\mathrm{Na}^{+}-\mathrm{Ca}^{2+}$ exchange, the less mobile $\mathrm{Ca}^{2+}$ ions occupied the stern layer and caused the decrease in $\sigma^{\prime \prime}$."

SC 6 - SIP spectra: Currently, the manuscript presents select spectra in the supporting information. The loam exhibits a clear sharp frequency peak, indicating a dominant polarization length-scale. Fitting, for example, a Cole-Cole model to the spectra could shed light on changes in polarization length scales and or ion mobilities that would help to improve the current conceptual model provided.

Autors' response - We accept this comment. The revised version of the manuscript will include a Cole-Cole model fit for obtained spectra to demonstrate the change in the polarization length scale as a result of the $\mathrm{Ca}^{2+}-\mathrm{Na}^{+}$exchange.

SC 7 - Figure 5c: The sand layer seems to be thin in relation to the loam layers, this is also mentioned in the text, line 210. Did the authors consider to what extent the signal in the sand-channel is influenced by the adjacent loam layers? Is the resolution of the effective electrode response volume fine enough to resolve a "sand-only" contribution? It would be good to provide a spectral comparison between the loam and the sand.

Printer-friendly version

Discussion paper

Autors' response - We agree that the electrodes measuring over the sandy 
part of the profile don't measure exclusively sand. For this reason, we refer to this layer as 'the sandy layer' and don't use the term 'sand only'. However, in our experimental setup the pairs of electrodes are fairly spaced and it is reasonable to assume that the sand layer is dominant in the signature captured by these electrodes. While this was not tested to the current geometry, it is clear (see for example Furman et al., 2003) that the region between the potential electrodes dominates the signal.

\section{Technical comments}

1. Line 4: change soil profiles to soil profile

Autors' response - Thank you for the attention. The sentence was corrected and now reads: "In this work...in homogeneous and heterogeneous soil profiles" (i.e the word 'a' was removed).

2. Line 46: What is the low frequency range?

Autors' response - We refer to the range of $1 \mathrm{mHz}$ to $1 \mathrm{kHz}$ as the low frequency range characteristic to IP. This is now specifically mentioned in the text.

3. Line 65: insert "a" in - and alternating current in "a" wide range of...

Autors' response - Corrected according to the comment.

4. How was the real conductivity normalized?

Printer-friendly version

Autors' response - The real conductivity was normalized to a $0-1$ scale according to the following equation: 


$$
\sigma_{\text {norm }}^{\prime}=\frac{\sigma^{\prime}-\sigma_{\min }^{\prime}}{\sigma_{\max }^{\prime}-\sigma_{\min }^{\prime}}
$$

5. What frequency is plotted in the time series plots? (This could be justified by presenting spectra)

Autors' response - All SIP time series figures are for a frequency of $1 \mathrm{~Hz}$. This is clearly mentioned in line 149 and in the captions of the figures.

\section{References:}

1. Izumoto, S., Huisman, J. A., Wu, Y., and Vereecken, H. (2020). Effect of solute concentration on the spectral induced polarization response of calcite precipitation. Geophysical Journal International, 220(2), 1187-1196.âÁ

2. Slater, L. D., DayâĂŘLewis, F. D., Ntarlagiannis, D., O'Brien, M., and Yee, N. (2009). Geoelectrical measurement and modeling of biogeochemical breakthrough behavior during microbial activity. Geophysical research letters, 36(14).âĂR

3. Mellage, A., Holmes, A. B., Linley, S., Vallee, L., Rezanezhad, F., Thomson, N., and Van Cappellen, P. (2018). Sensing coated iron-oxide nanoparticles with spectral induced polarization (SIP): experiments in natural sand packed flow-through columns. Environmental science and technology, 52(24), 14256-14265.âĂŔ

4. Leroy, P., and Revil, A. (2009). A mechanistic model for the spectral induced polarization of clay materials. Journal of Geophysical Research: Solid Earth, 114(B10).âÁR 5. Schwartz, N., Huisman, J. A., and Furman, A. (2012). The effect of NAPL on the electrical properties of unsaturated porous media. Geophysical Journal International, 188(3), 1007-1011.

âĂŔ 6. Vaudelet, P., Revil, A., Schmutz, M., Franceschi, M., and Bégassat, P. (2011). Induced polarization signatures of catins exhibiting differential sorption behaviors in 
saturated sands. Water Resources Research, 47(2).

7. Furman, A., Ferré, T. P., and Warrick, A. W. (2003). A sensitivity analysis of electrical resistivity tomography array types using analytical element modeling. Vadose Zone Journal, 2(3), 416-423.âĂRâĂŔ âĂŔ

Interactive

comment 